\title{
Comparison of free-electron laser amplifiers based on a step-tapered optical klystron and a conventional tapered wiggler
}

\author{
H.P. Freund \\ Los Alamos National Laboratory, Los Alamos, New Mexico 87545, USA
}

(Received 2 April 2013; published 4 June 2013)

\begin{abstract}
Free-electron laser amplifiers have been operated at high efficiency at wavelengths from the microwave through the visible. Typically, these amplifiers require long tapered sections and produce spent beams with large energy spreads that are 4-5 times the electronic efficiency. In addition, while optical guiding during exponential growth in the uniform wiggler section confines the optical mode, the guiding disappears in the tapered wiggler section resulting in a relatively large optical mode at the wiggler exit. Optical klystrons consist of a Modulator wiggler that induces a velocity modulation on the electron beam followed by a magnetic dispersive section that enhances the velocity modulation prior to injection into a second, radiator wiggler. Optical klystrons have been operated over a broad spectral range; however, no optical klystron has been built with a tapered radiator wiggler. A comparison between a optical klystron with a step-tapered Radiator wiggler and a conventional tapered wiggler amplifier is analyzed in this paper. The purpose of the step taper is to both enhance the efficiency and to extend the range of the exponential gain and so preserve the optical guiding over a longer interaction length. The step-tapered optical klystron and a tapered wiggler amplifier are compared for a nominal set of parameters to determine the differences in the efficiency, interaction length, spent beam energy spread, and the size of the optical mode at the wiggler exit.
\end{abstract}

\section{INTRODUCTION}

Free-electron lasers (FELs) have been designed to operate over virtually the entire electromagnetic spectrum [1] from microwaves through $\mathrm{x}$ rays and in a variety of configurations including amplifiers and oscillators. Tapered wiggler FEL amplifiers have demonstrated efficiencies as high as $35 \%$ at a wavelength of $8 \mathrm{~mm}$ [2], and about $1 \%$ at a wavelength of $800 \mathrm{~nm}$ [3]. Since the tapered section must start slightly prior to the saturation point of the uniform wiggler, this means that the overall wiggler length can be large. Further, the optical mode is guided only within the uniform wiggler where the mode grows exponentially. Once the tapered wiggler section begins, the mode undergoes near-free space diffraction and, so, can expand to a relatively large size at the wiggler exit. Finally, the full energy spread of the spent beam can be as much as 4-5 times the efficiency, and this can hinder energy recovery schemes. In view of these properties of the tapered wiggler FEL amplifier, a step-tapered optical klystron may be an attractive alternative design.

Optical klystrons have been in use for decades and the first use was in an ultraviolet FEL oscillator [4]. An optical klystron (OK), as shown schematically in Fig. 1, is fundamentally composed of a Modulator wiggler that imposes a velocity modulation on the electrons followed by a

Published by the American Physical Society under the terms of the Creative Commons Attribution 3.0 License. Further distribution of this work must maintain attribution to the author(s) and the published article's title, journal citation, and DOI. magnetic dispersive section that enhances the modulation prior to injection into a Radiator wiggler that takes the interaction with the modulation-enhanced electrons to saturation. The magnetic dispersive element is, typically, a three- or four-dipole chicane. The Radiator can be tuned to the fundamental or a harmonic of the Modulator, in which case the interaction is referred to as high-gain harmonic generation $[5,6]$.

The OK has several advantages over a conventional wiggler. In a conventional wiggler, the interaction between the electrons and the optical modes results in a density and velocity modulation that increases exponentially as the interaction proceeds. The velocity modulation, in particular, is associated with an increasing energy spread over the course of the wiggler. This increasing energy spread results in a degradation of the resonant interaction and can limit the extraction efficiency. In contrast, the enhanced bunching in the chicane can occur faster than the increasing modulation in the wiggler, thereby shortening the interaction length. In addition, the enhanced bunching does not result in an increased energy spread. In principle, therefore, the OK can result in both a shorter interaction length and a higher extraction efficiency over a conventional uniform wiggler. In this paper, the OK concept is extended to

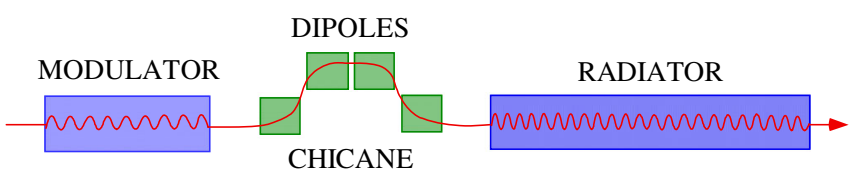

FIG. 1. Schematic representation of an optical klystron. 
include a step-tapered Radiator wiggler for enhanced efficiency. The step taper has been discussed in the literature for high-power FEL amplifier operation [7], although this is the first study of its use with an optical klystron. The purpose of the step taper is to extend the regime of exponential growth where the mode is optically guided. In this way, the mode size at the wiggler exit can be reduced relative to that found in a conventionally tapered wiggler. In addition, the slippage of the optical pulse over the electron beam is reduced in the exponential gain regime due to a reduction in the group velocity [8], so that the extension of exponential gain in the step taper acts to preserve the overlap of the optical mode and the electron bunch over an extended interaction length.

Three-dimensional, time-dependent simulations of an infrared, step-tapered OK and a comparable conventional tapered wiggler amplifier are presented using the MEDUSA simulation code. The numerical analysis is performed for a nominal set of beam, wiggler, and optical parameters, and the results for the step-tapered OK are compared with those for a conventional tapered wiggler FEL amplifier.

\section{THE NUMERICAL FORMULATION}

The numerical simulation of the step-tapered optical klystron is conducted using the MEDUSA code. MEDUSA is a three-dimensional simulation code that includes time dependence, harmonics, and start-up from noise [9-12]. It models helical and planar wigglers and the optical field is represented as a superposition of Gaussian modes using an adaptive eigenmode expansion that tracks the mode size and phase front curvature based upon the interaction [13]. No wiggler average orbit analysis is used and electron trajectories are integrated using the three-dimensional Lorentz force equations in the combined magnetostatic and optical fields. Models for both quadrupoles and dipoles are included. As such, MEDUSA copropagates the electrons and the optical field self-consistently through the wigglers, drift space, and either dipoles (i.e., the chicane) or quadrupoles. The time dependence is treated in either of two ways. First, the electron beam and the optical mode are described by an ensemble of temporal slices where each slice is advanced from $z \rightarrow z+\Delta z$ as in steady-state simulations, after which the field is allowed to slip relative to the electrons. Second, an explicit polychromatic expansion of the fields can be employed. These two algorithms are equivalent [14]; however, the former is simpler to employ and is used here. Note that the first time-dependence algorithm can be combined with a polychromatic harmonic representation to treat the evolution of the fundamental and harmonics in the time domain.

\section{THE NUMERICAL ANALYSIS}

The parameters for the nominal case that is under consideration are shown in Table I for the optical klystron.
TABLE I. Nominal beam, wiggler, chicane, and optical parameters under consideration.

\begin{tabular}{ll}
\hline \hline Electron beam & \\
Electron energy & $85.25 \mathrm{MeV}$ \\
Bunch charge & $650 \mathrm{pC}$ \\
Bunch duration & $1.0 \mathrm{psec}$ \\
Normalized emittance & $10-15 \mu \mathrm{m}$ \\
Energy spread & $0.1-0.3 \%$ \\
Modulator/radiator & Parabolic-pole-face \\
Modulator/radiator period & $3.0 \mathrm{~cm}$ \\
Modulator length & $1.77 \mathrm{~m}$ \\
Modulator amplitude & $5.0 \mathrm{kG}$ \\
Initial radiator amplitude & $5.0 \mathrm{kG}$ \\
Wiggler separation & $0.51 \mathrm{~m}$ \\
Chicane & $4 \mathrm{dipoles}$ (hard edge) \\
Dipole length & $0.09 \mathrm{~m}$ \\
Dipole gaps & $0.03 \mathrm{~m}$ \\
Overall chicane length & $0.45 \mathrm{~m}$ \\
Chicane field & $2-3 \mathrm{kG}$ \\
Optical field & Parabolic pulse \\
Seed wavelength & $1.06 \mathrm{microns}$ \\
Seed power & $1.0 \mathrm{~kW}$ \\
Seed duration & $1.0 \mathrm{psec}$ \\
\hline \hline
\end{tabular}

The conventional tapered wiggler amplifier uses the same beam and optical parameters, but the wiggler is lengthened. The electron beam has an energy of $82.25 \mathrm{MeV}$ and a bunch charge of $650 \mathrm{pC}$. The pulse shape is assumed to be parabolic with a full width of 1.0 psec. The normalized emittance ranges over $10-15 \mu \mathrm{m}$ and the rms energy spread over $0.1 \%-0.3 \%$. The Radiator and Modulator wigglers are assumed to provide weak focusing with parabolic pole faces, and the electron beam is injected with the matched-beam radius. The period of both the Radiator and Modulator is $3.0 \mathrm{~cm}$, and the amplitude of the Modulator is $5.0 \mathrm{kG}$ over a length of $1.77 \mathrm{~m}$. This represents a total of 59 periods and, since MEDUSA simulates the entry and exit from the wiggler, the first and last periods describe an up and a down taper for the field. Hence, the Modulator is characterized by 57 periods with a uniform field strength. The radiator has an initial amplitude of $5.0 \mathrm{kG}$, and we optimize the step taper and overall length for maximum output. The chicane is composed of four hard-edge dipoles each of which is $0.09 \mathrm{~m}$ in length and which are separated by gaps of $0.03 \mathrm{~m}$. This results in an overall chicane length of $0.45 \mathrm{~m}$, and the dipole fields are varied between $2-3 \mathrm{kG}$. The gaps between the chicane and the wigglers are also assumed to be $0.03 \mathrm{~m}$, so that the overall gap between the Modulator and Radiator is $0.51 \mathrm{~m}$ long. The OK is tuned to a wavelength of $1.06 \mu \mathrm{m}$ and the seed laser is assumed to provide a parabolic pulse with a full width of 1.0 psec to match the electron bunch and with a peak power of $1.0 \mathrm{~kW}$ corresponding to a pulse energy of about $0.67 \mathrm{~nJ}$. 


\section{A. Comparison between the optical klystron and a uniform wiggler}

The chicane (dipole fields) must be optimized before proceeding with the tapered Radiator. The optimal dipole fields are dependent upon the modulation induced on the electron bunch due to the interaction in the Modulator and this, in turn, depends upon the beam parameters, the Modulator, and the seed power.

It should be noted that the OK is sensitive to the energy spread. The ratio of the path length, $L$, in the chicane to the wavelength, $\lambda$, is

$$
\frac{L}{\lambda}=\frac{2}{3} \frac{L_{d}^{3} \Omega_{0}^{2}}{\gamma^{2} c^{2} \lambda}
$$

where $L_{d}$ is the dipole length, $\Omega_{0}$ is the dipole cyclotron frequency, $\gamma$ is the relativistic factor, and $c$ is the speed of light in vacuo. As a result, the change in path length due to a change in the energy is

$$
\frac{\Delta L}{\lambda}=\frac{4}{3} \frac{L_{d}^{3} \Omega_{0}^{2}}{\gamma^{2} c^{2} \lambda} \frac{\Delta \gamma}{\gamma} .
$$

The change in path length must be comparable to or less than the wavelength in order for efficient bunching to occur. For the present parameters, therefore, we have that $|\Delta L / \lambda| \approx 475|\Delta \gamma / \gamma|$ for a $2.0 \mathrm{kG}$ dipole field which means that the energy spread must be on the order of about $0.2 \%$ or less.

The evolution of the optical pulse for an emittance of $10 \mu \mathrm{m}$ and energy spreads of $0.1 \%, 0.2 \%$, and $0.3 \%$ is shown in Fig. 2 where the optimal choices of the dipole fields $(2.4-2.5 \mathrm{kG})$ are indicated. It is evident that the optical field grows exponentially in the Modulator indicating that velocity modulation is taking place. The growth of the optical field ceases in the gap/chicane between the wigglers. The enhanced bunching in the chicane preconditions the field for rapid growth in the Radiator and

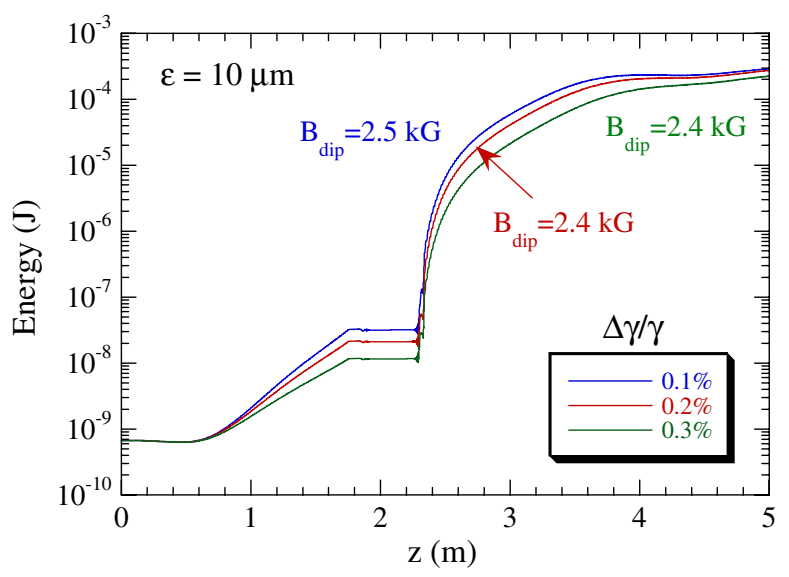

FIG. 2. Evolution of the optical pulse for an emittance of $10 \mu \mathrm{m}$ and energy spreads of $0.1 \%, 0.2 \%$, and $0.3 \%$ for the optimal dipole fields. saturation is found over an additional length of about $1.8 \mathrm{~m}$. The overall distance to saturation is about $3.8 \mathrm{~m}$. The pulse energy at saturation decreases with increasing energy spread as expected. The saturated pulse energy is $0.24 \mathrm{~mJ}$ at an energy spread of $0.1 \%$, which decreases slightly to $0.21 \mathrm{~mJ}$ as the energy spread increases to $0.2 \%$. As expected on the basis of the path length argument above, the saturated pulse energy decreases more dramatically to $0.15 \mathrm{~mJ}$ as the energy spread increases to $0.3 \%$.

The phase space dynamics in the chicane are illustrated in Figs. 3 and 4, where $\psi$ denotes the ponderomotive phase, which show the phase space at the entrance to the chicane (Fig. 3) and at the exit from the chicane (Fig. 4) for an emittance of $10 \mu \mathrm{m}$, an energy spread of $0.1 \%$, and the optimal dipole field of $2.5 \mathrm{kG}$. As shown in Fig. 3, the phase space for a beamlet that is one wavelength long exhibits some dispersion as well as a modulation due to the interaction in the Modulator. The effect of the chicane is dramatic, as shown in Fig. 4, where (1) substantial dispersion is found as the original beamlet is now smeared over a range of phases of about $-522<\psi / 2 \pi<-514$

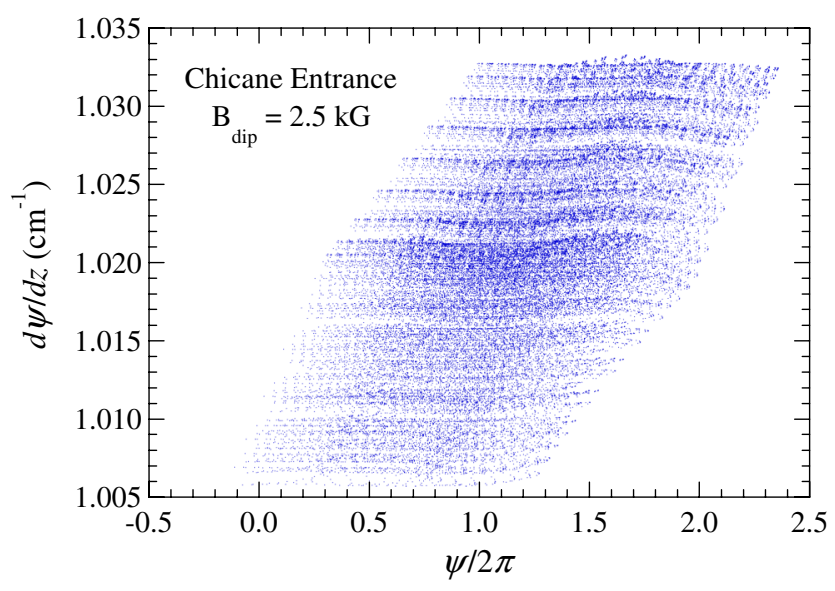

FIG. 3. Phase space at the entrance to the chicane.

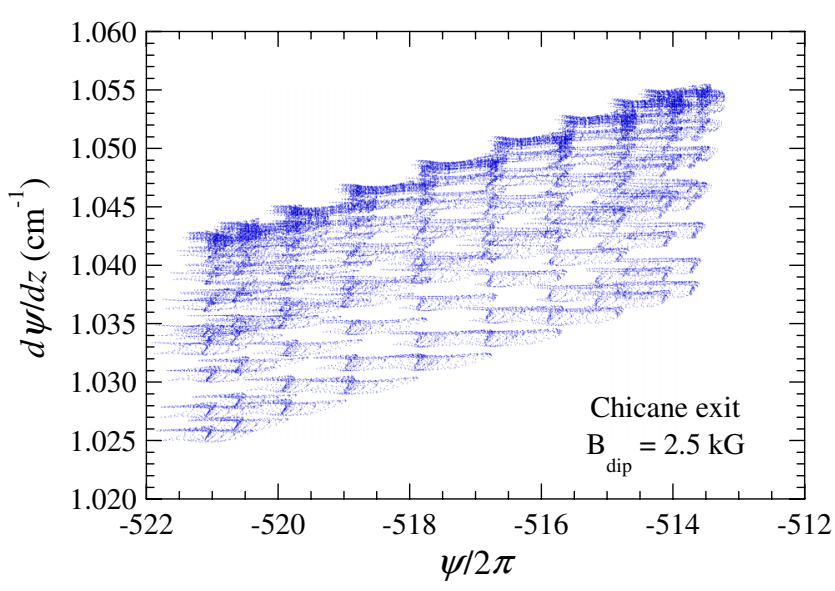

FIG. 4. Phase space at the exit from the chicane. 
corresponding to a width of about eight wavelengths, and (2) pronounced bunching is exhibited every wavelength. The dispersion is due to the variation in the axial velocity of the beam as it traverses the chicane. It is found that $\langle d \psi / d z\rangle \approx-64 \mathrm{~cm}^{-1}$ while $(d \psi / d z)_{\mathrm{rms}} \approx-92 \mathrm{~cm}^{-1}$ through the chicane. Over the entire chicane $(45 \mathrm{~cm})$, therefore, the phase will change by about $460 \pi-660 \pi$, which is in accord with the simulation. Further, at any given point in the chicane, $d \psi / d z$ spans a range of about $2 \%$ of $\langle d \psi / d z\rangle$, which corresponds to a spread in the phase space of about 8-9 wavelengths, which is also in accord with the simulation. It is this bunching that drives the amplification in the Radiator so strongly.

The interaction is degraded as the emittance increases, but is still relatively strong for an emittance of $15 \mu \mathrm{m}$. The amplification of the seed pulse for an emittance of $15 \mu \mathrm{m}$ and energy spreads of $0.1 \%, 0.2 \%$, and $0.3 \%$ is shown in Fig. 5, where strong amplification is still found. However, the interaction is weaker than what was found at $10 \mu \mathrm{m}$ as the overall saturation distance has increased to about $5.1 \mathrm{~m}$ and the saturated pulse energies have dropped to $0.21,0.18$, and $0.13 \mathrm{~mJ}$ for energy spreads of $0.1 \%, 0.2 \%$, and $0.3 \%$, respectively.

A comparison between the $\mathrm{OK}$ and a single wiggler is shown in Figs. 6-8 for an emittance of $10 \mu \mathrm{m}$ and energy spreads of $0.1 \%, 0.2 \%$, and $0.3 \%$, respectively. The optimization procedure has been well established over a long history of tapered wiggler studies and designs. The optimal start-taper point corresponds to the point at which the electrons start to cross the separatrix into the trappedparticle regime. The optimal taper slope describes a balance between the shift in the resonance condition due to the decreasing electron energy and the rate at which energy is extracted from the electrons. If the slope is too gradual (sharp), then the electrons cannot remain in balance with changing resonance condition. In practice, the optimal start-taper point and slope are found by performing a range of simulations.

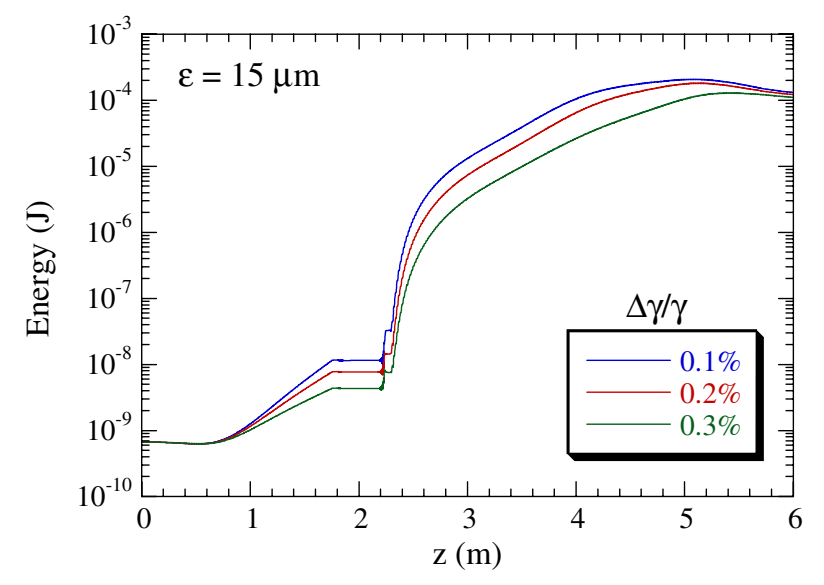

FIG. 5. Evolution of the optical pulse for an emittance of $15 \mu \mathrm{m}$ and energy spreads of $0.1 \%, 0.2 \%$, and $0.3 \%$ for the optimal dipole fields.

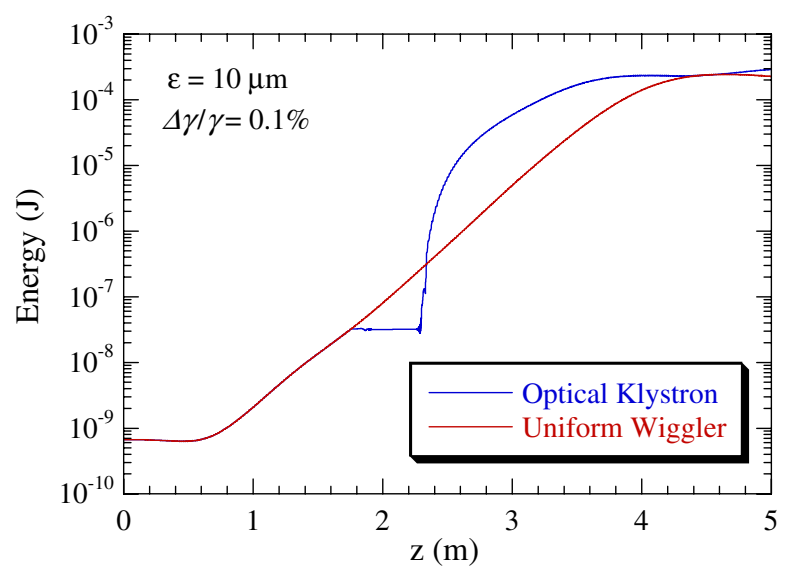

FIG. 6. Comparison between the $\mathrm{OK}$ and a single wiggler for a $10 \mu \mathrm{m}$ emittance and an energy spread of $0.1 \%$.

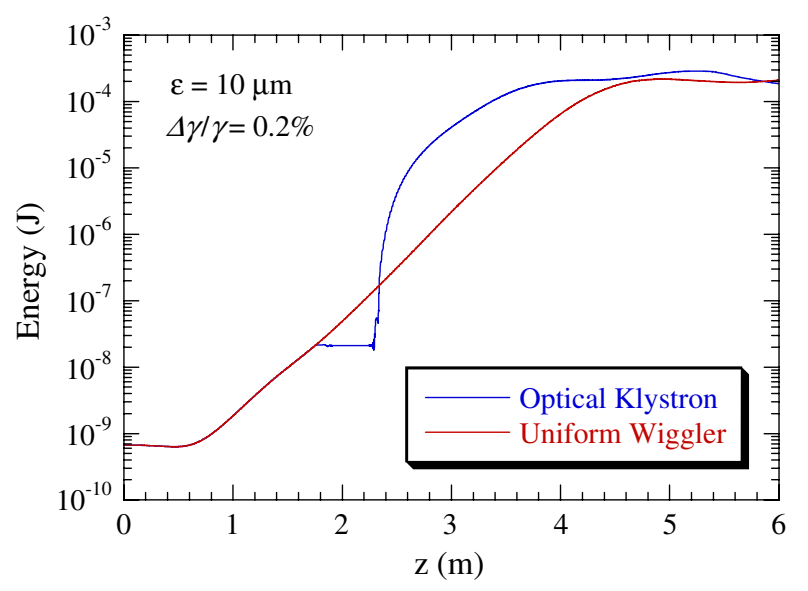

FIG. 7. Comparison between the $\mathrm{OK}$ and a single wiggler for a $10 \mu \mathrm{m}$ emittance and an energy spread of $0.2 \%$.

In the case of $0.1 \%$ energy spread, the two cases reach similar extraction efficiencies but the OK reaches saturation over a shorter overall length by approximately $0.6 \mathrm{~m}$. The advantage in saturation distance for the $\mathrm{OK}$ is retained

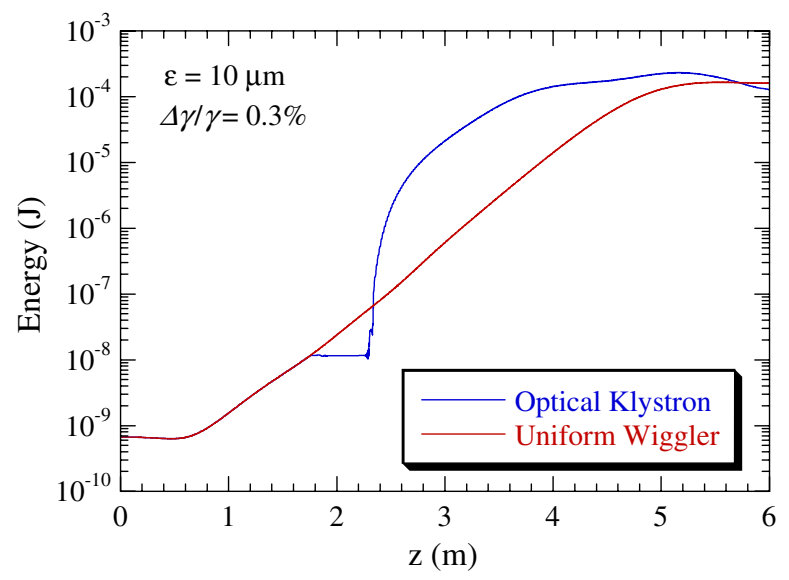

FIG. 8. Comparison between the $\mathrm{OK}$ and a single wiggler for a $10 \mu \mathrm{m}$ emittance and $\Delta \gamma / \gamma=0.3 \%$. 
as the energy spread increases to $0.2 \%$ (see Fig. 7). As discussed previously, coherent bunching in the chicane begins to fail as the energy spread increases to $0.3 \%$ and the performance of the OK is noticeably degraded. However, as shown in Fig. 8, the performance of the single wiggler is degraded even more than the OK, which now shows both a shorter saturation distance and a higher extraction efficiency.

\section{B. Comparison between a step-tapered optical klystron and a tapered wiggler}

The Radiator in a step-tapered OK has been optimized over an overall length of $5.6 \mathrm{~m}$ using four steps. A summary of the steps is shown in Table II, including the Modulator as a first step. The evolution of the pulse energy in the optimized OK configuration (blue) as well as the optimized conventional tapered wiggler (red) is shown in Fig. 9, where the positions and amplitudes of the several steps in the wiggler in the OK are clearly shown. Saturation is found with a pulse energy of $1.06 \mathrm{~mJ}$ in the middle of the last step of the Radiator for an overall length of $5.6 \mathrm{~m}$. This represents an extraction efficiency of about $2.0 \%$. Optimization of the tapered wiggler requires that the start-taper point must be selected as the electrons start to become trapped in the ponderomotive wave but before they undergo one synchrotron oscillation. The taper slope must be chosen to balance the energy loss of the electrons by the resonance change in the decreasing wiggler field. If the downward slope is too gradual, then the resonance cannot be maintained. In contrast, if the slope is too extreme, then the electrons slosh out of the trough of the ponderomotive wave and the interaction suffers. For the case of interest, the optimal start-taper point is at a distance of $4.1 \mathrm{~m}$ downstream from the wiggler entrance with a slope of $-314 \mathrm{G} / \mathrm{m}$. Using these optimized taper parameters, the pulse energy reaches the same level as for the steptapered OK of about $1.06 \mathrm{~mJ}$ after $6.62 \mathrm{~m}$. It should be noted that the slope of the amplification is falling rapidly at this point and the tapered wiggler interaction is nearing its practical limit.

Optical guiding in the step-tapered OK differs from that in the long, tapered wiggler. To illustrate this, we consider the peak in the output pulses for each case. The evolution of the optical mode size for the step-tapered OK (blue) and

TABLE II. Summary of the steps in the OK wiggler. Note that step 1 denotes the Modulator.

\begin{tabular}{lccc}
\hline \hline Step & Wiggler field & Start position & Stop position \\
\hline 1 & $5.0 \mathrm{kG}$ & $0.0 \mathrm{~m}$ & $1.77 \mathrm{~m}$ \\
2 & $5.0 \mathrm{kG}$ & $2.28 \mathrm{~m}$ & $3.45 \mathrm{~m}$ \\
3 & $4.9 \mathrm{kG}$ & $3.45 \mathrm{~m}$ & $4.26 \mathrm{~m}$ \\
4 & $4.7 \mathrm{kG}$ & $4.26 \mathrm{~m}$ & $4.98 \mathrm{~m}$ \\
5 & $4.4 \mathrm{kG}$ & $4.98 \mathrm{~m}$ & $6.00 \mathrm{~m}$ \\
\hline \hline
\end{tabular}

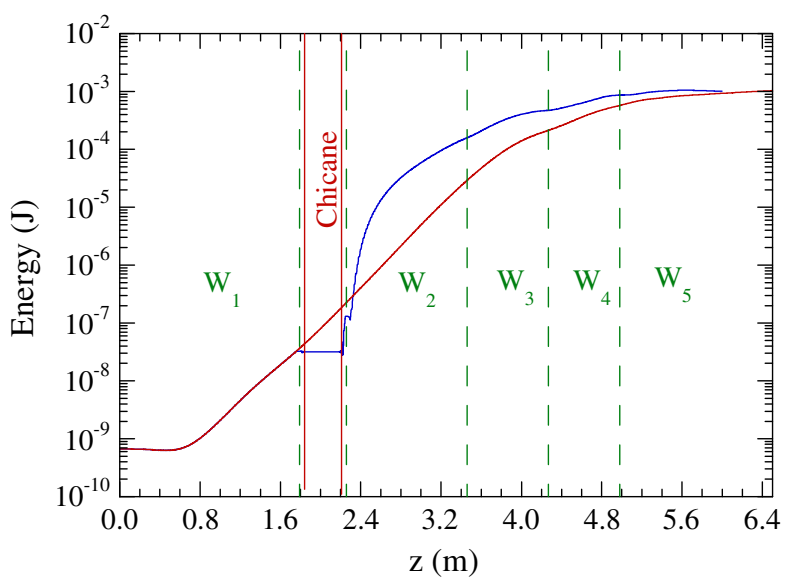

FIG. 9. Evolution of the pulse energy for the optimized steptapered OK (blue) and the optimized conventional tapered wiggler (red).

the conventional tapered wiggler (red) is shown in Fig. 10. Since exponential gain is maintained over much of the Modulator and Radiator, and only ceases near saturation in the last step of the Modulator, we expect that the optical mode will be guided throughout most of the Radiator. This is indeed what is shown in Fig. 10. The mode size increases initially in the Modulator for several gain lengths until exponential growth begins, after which it is rapidly focused down to a spot size of about $0.045 \mathrm{~cm}$. The mode expands in the gap and chicane between the wigglers where the interaction ceases, but is then focused rapidly to a spot size of about $0.045 \mathrm{~cm}$ once the electron bunch and optical mode enters the Radiator. This spot size is maintained over much of the radiator until the nonlinear phase of the interaction near saturation begins near the middle of the last step. The spot size at saturation (i.e., $z=5.6 \mathrm{~m}$ ) is about $0.056 \mathrm{~cm}$. This is in sharp contrast to the expansion of the optical pulse in the long, tapered wiggler. In this case, as in the case of the step-tapered OK, the mode

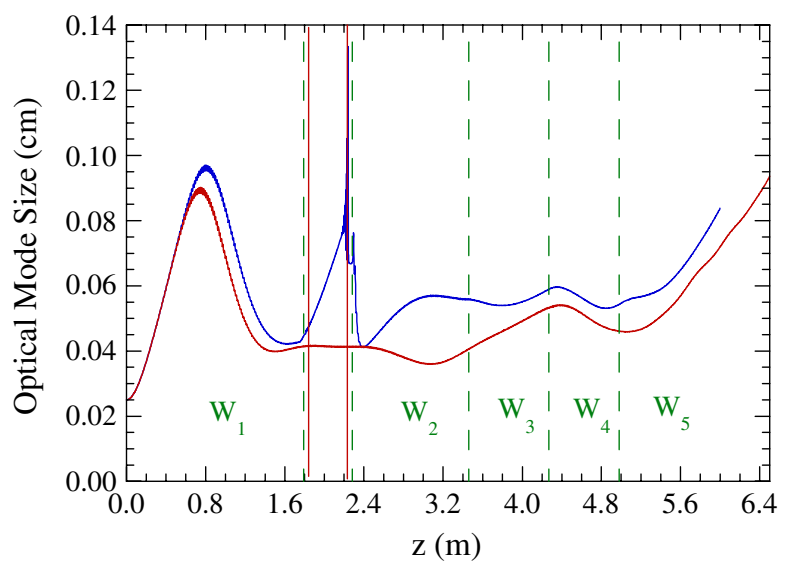

FIG. 10. Evolution of the optical mode size at the peak of the optical pulse for the step-tapered OK (blue) and the conventional tapered wiggler (red). 
initially expands after the wiggler entrance, but is focused down as the exponential gain begins. However, the guiding of the optical mode largely ceases when the taper begins at $z=4.1 \mathrm{~m}$, after which near-free-space diffraction takes over and the mode size increases to about $0.10 \mathrm{~cm}$ at $6.62 \mathrm{~m}$. This is almost twice the size of the output pulse in the step-tapered OK. It should be remarked that the mode expansion in the long, tapered wiggler limits the interaction efficiency because as the mode expands the overlap between the optical mode and the electron bunch (the so-called filling factor) decreases and progressively less of the optical mode participates in the interaction.

The modal decomposition of the optical pulses for the step-tapered $\mathrm{OK}$ and the conventional tapered wiggler amplifier at the positions where the extraction efficiency is $2 \%$ (i.e., $z=5.6 \mathrm{~m}$ for the step-tapered OK and $6.62 \mathrm{~m}$ for the tapered wiggler amplifier) are shown in Table III. In the case of the step-tapered OK, approximately $75 \%$ of the power is carried by the $\mathrm{TEM}_{00}$ mode with another $20.1 \%$ carried by the $\mathrm{TEM}_{20}$ and $\mathrm{TEM}_{02}$ modes. In the case of the tapered wiggler amplifier, $82.4 \%$ is carried by the $\mathrm{TEM}_{00}$ mode while the $\mathrm{TEM}_{20}$ and $\mathrm{TEM}_{02}$ account for another $16.4 \%$. The transverse mode structures for the two configurations are shown in Figs. 11 and 12, respectively, for the step-tapered OK and the tapered wiggler amplifier, where the amplitude is plotted versus the $x$ and $y$ dimensions normalized to the mode waist size. It should be remarked that the Rayleigh range for the step-tapered OK is much smaller than that for the tapered wiggler amplifier because the optical mode size is so much smaller for this configuration; hence, the transverse expansion in free space for the step-tapered OK will be much more rapid than for the tapered wiggler amplifier.

Since the comparison between the two configurations is made for comparable extraction efficiencies, and since it is well known that the spent beam energy spread in an FEL [1] is comparable to the extraction efficiency, the two configurations result in very similar spent beam distributions as shown in Figs. 13 and 14. The rms energy spreads for the spent beams are $2.2 \%$ and $2.5 \%$ for the step-tapered $\mathrm{OK}$ and the tapered wiggler amplifier, respectively.

The optical pulse shape after $5.6 \mathrm{~m}$ in the step-tapered OK (blue) and after $6.62 \mathrm{~m}$ in the conventional tapered wiggler

TABLE III. Modal decomposition at the output for the steptapered $\mathrm{OK}$ and the tapered wiggler amplifier.

\begin{tabular}{lcc}
\hline \hline Mode & Step-tapered OK & Tapered wiggler \\
\hline $\mathrm{TEM}_{00}$ & $74.5 \%$ & $82.4 \%$ \\
$\mathrm{TEM}_{20}$ & $8.6 \%$ & $3.4 \%$ \\
$\mathrm{TEM}_{02}$ & $11.5 \%$ & $13.0 \%$ \\
$\mathrm{TEM}_{22}$ & $1.2 \%$ & $0.6 \%$ \\
$\mathrm{TEM}_{04}$ & $1.7 \%$ & $0.1 \%$ \\
$\mathrm{TEM}_{40}$ & $2.5 \%$ & $0.5 \%$ \\
\hline \hline
\end{tabular}

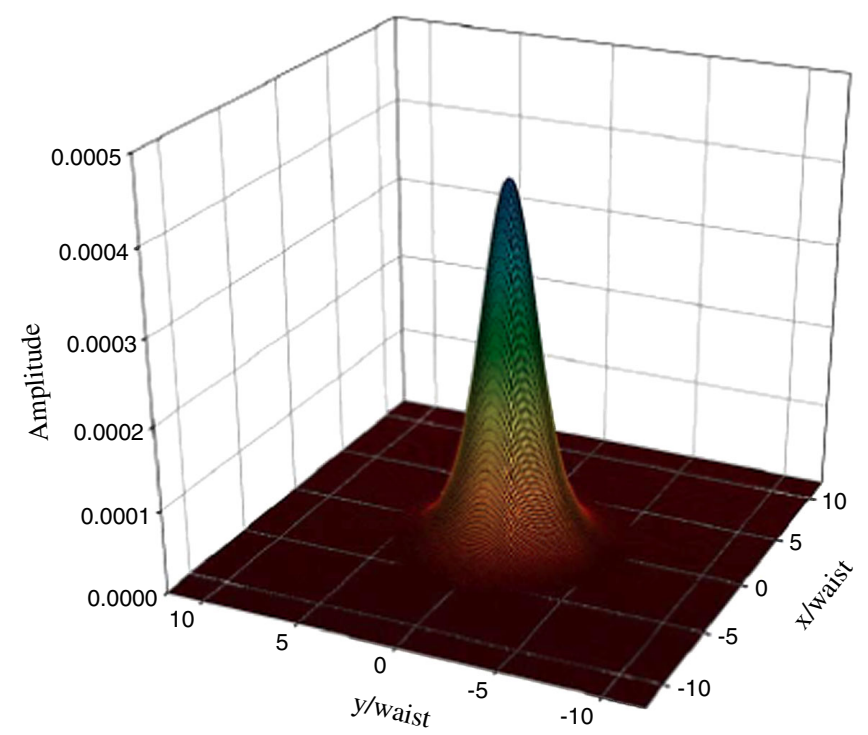

FIG. 11. Transverse mode structure for the step-tapered OK.

(red) is shown in Fig. 15. The electron bunch is shown using the shaded area in the figure. Since the bunch shape is parabolic, the peak in the current profile is at the center of the time window (i.e., $1.0 \mathrm{psec}$ ). The initial optical seed pulse was also assumed to be parabolic with a full width of $1.0 \mathrm{psec}$. At saturation the optical pulse has become elongated relative to its initial shape and has slipped ahead of the electron bunch by about $0.24 \mathrm{psec}$. The usual measure of slippage is that the optical pulse slips ahead of the electrons by one wavelength per wiggler period. Ignoring the chicane, this would imply that the optical pulse slips ahead of the electron bunch by about $0.61 \mathrm{psec}$ over the $5.6 \mathrm{~m}$ of the interaction. However, the reduction in the group velocity in

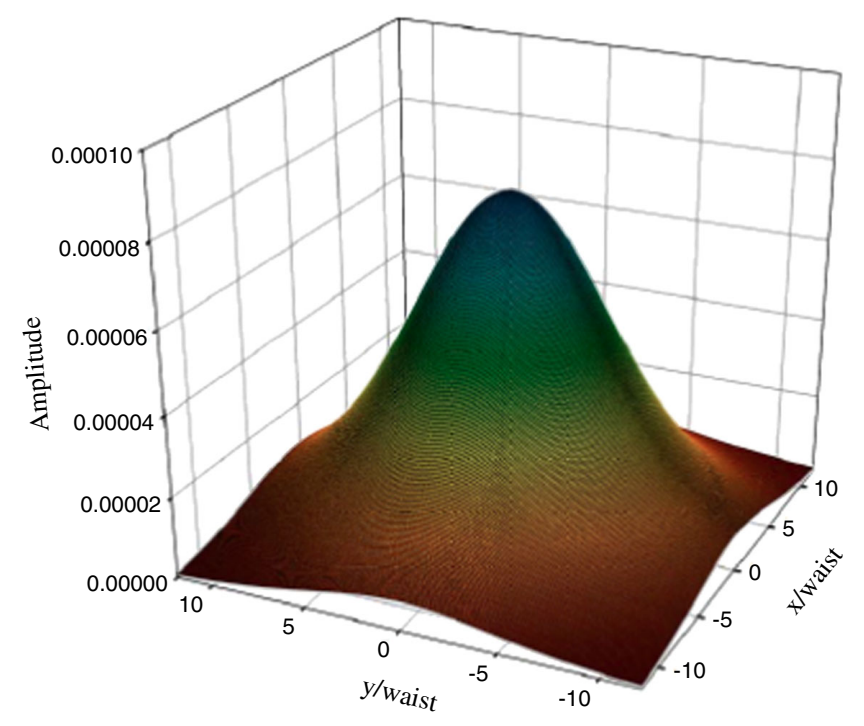

FIG. 12. Transverse mode structure for the tapered wiggler amplifier. 


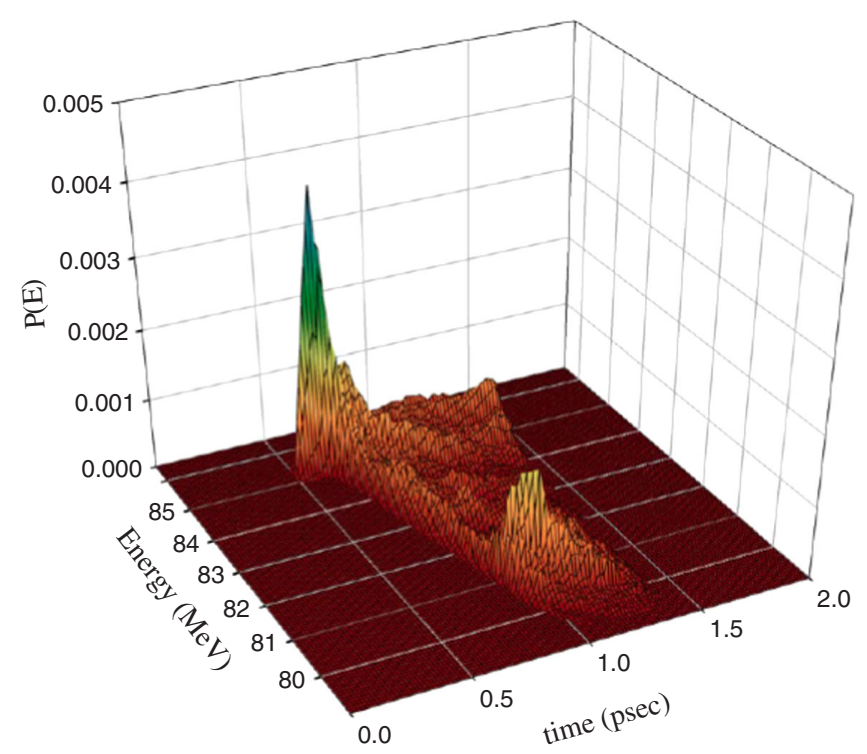

FIG. 13. The spent beam energy distribution for the steptapered OK.

the exponential gain regime [7] results in a reduction of slippage by one-third. Since the step taper preserves the exponential gain over much of the interaction region, this would imply that the optical pulse should slip ahead by about $0.20 \mathrm{psec}$, which is in rough agreement with the simulation. The optical pulse shape after $6.62 \mathrm{~m}$ of the tapered wiggler amplifier simulation is shown in red in the figure. Observe that the pulse is comparable to that for the step-tapered OK but that the peak has slipped farther ahead of the electron bunch. In this case, the peak of the optical pulse has slipped ahead by about $0.3 \mathrm{psec}$. In view of

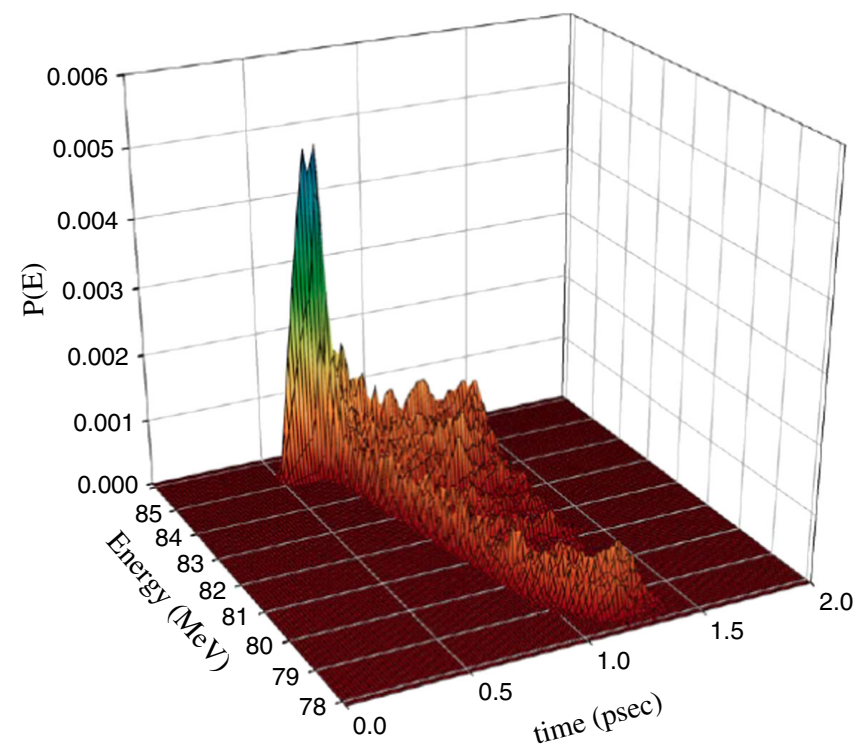

FIG. 14. Spent beam energy distribution for the tapered wiggler amplifier.

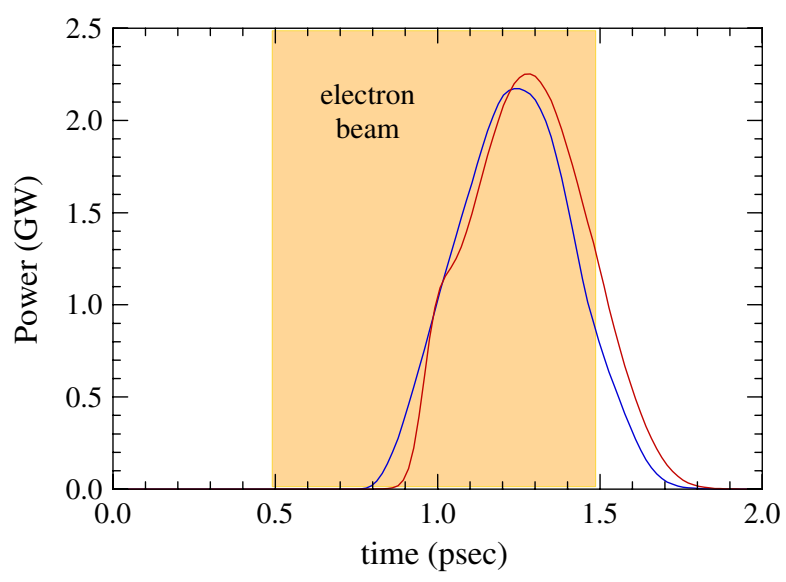

FIG. 15. Optical pulse shapes at saturation for the OK (blue) and the conventional tapered wiggler (red) and showing the relative position of the electron beam in the shaded area.

the fact that slippage is reduced by about one-third in the first $4.1 \mathrm{~m}$ of the wiggler prior to the start of the taper, it is expected that the optical pulse should slip ahead of the electron bunch by about 0.46 psec over the $6.62 \mathrm{~m}$ of wiggler. While this is not a great discrepancy, it should be noted that as the optical pulse slips ahead of the electron bunch in the long wiggler, it experiences a rapidly declining current that limits the amplification of the mode. As a result, the peak in the optical pulse will slip ahead of the electron bunch more slowly than the simple slippage estimate would predict. It should be noted that the step taper could also be used independently of the OK and this would have limited slippage further; however, this is beyond the scope of this paper.

The disparate temporal structures of the optical pulses in the step-tapered OK and the tapered wiggler have implications for the output spectra from the two configurations. The output spectrum from the step-tapered OK (blue) and the conventional tapered wiggler (red) is shown in Fig. 16.

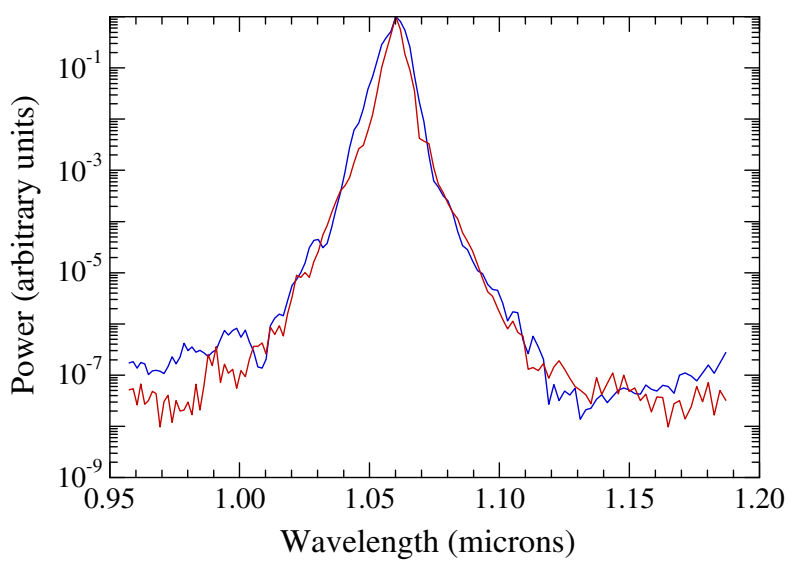

FIG. 16. Output spectrum from the step-tapered OK (blue) and the conventional tapered wiggler (red). 
The peak for both configurations is clearly at $1.06 \mu \mathrm{m}$ corresponding to the seed laser. The full width half maximum (FWHM) linewidth of the pulse at $z=5.60 \mathrm{~m}$ for the step-tapered OK is about $0.006 \mu \mathrm{m}$ so that $\Delta \lambda / \lambda \approx$ $0.57 \%$. The FWHM linewidth for the conventional tapered wiggler at $z=6.62 \mathrm{~m}$ is about $0.004 \mu \mathrm{m}$ so that $\Delta \lambda / \lambda \approx$ $0.38 \%$. These results are comparable, although the tapered wiggler amplifier has a somewhat narrower linewidth.

\section{SUMMARY AND DISCUSSION}

In this paper, a comparison between an infrared, steptapered OK and a conventional tapered wiggler amplifier has been presented for a nominal set of beam, wiggler, chicane, and optical parameters relevant to infrared FELs. The results indicate that the step-tapered OK can produce high extraction efficiencies. In comparison with the tapered wiggler amplifier, the step-tapered OK has a significantly shorter interaction length and produces a more compact optical mode at the wiggler exit than a tapered wiggler amplifier, which translates into a more rapidly expanding optical beam into free space. However, the two configurations result in comparable spent beam energy spreads and optical linewidths. Since slippage is reduced in the step-tapered OK due to the shorter interaction length and the use of a step taper it may be possible to increase the saturation efficiency further than has been examined in this paper. As a result, the step-tapered OK can provide an alternative path to the design of an FEL amplifier. While the present results describe only a specific infrared design, the results are generally applicable over a wider spectral range. In particular, the finding that the $\mathrm{OK}$ is no more sensitive to energy spread than a conventional wiggler design may have implications for short wavelength operation with multistage OKs [15-18].

\section{ACKNOWLEDGMENTS}

This work was supported by the Office of Naval Research and the Joint Technology Office.
[1] H.P. Freund and T.M. Antonsen, Jr., Principles of Free-Electron Lasers (Chapman \& Hall, London, 1996), 2nd ed.

[2] T. J. Orzechowski, B.R. Anderson, J.C. Clark, W.M. Fawley, A. C. Paul, D. Prosnitz, E. T. Scharlemann, S. M. Yarema, D. B. Hopkins, A. M. Sessler, and J. S. Wurtele, Phys. Rev. Lett. 57, 2172 (1986).

[3] X. J. Wang, H. P. Freund, D. Harder, W. H. Miner, Jr., J. B. Murphy, H. Qian, Y. Shen, and X. Yang, Phys. Rev. Lett. 103, 154801 (2009).

[4] G. N. Kuliapanov, V.N. Litvinenko, I. V. Panaev, V.M. Popik, A.N. Skrinsky, A. S. Sokolov, and N. A. Vinokurov, Nucl. Instrum. Methods Phys. Res., Sect. A 296, 1 (1990).

[5] L. H. Yu et al., Science 289, 932 (2000).

[6] A. Doyuran, M. Babzien, T. Shaftan, L. H. Yu, L.F. Dimauro, I. Ben-Zvi, S.G. Biedron, W. Graves, E. Johnson, S. Krinsky, R. Malone, I. Pogorelsky, J. Skaritka, G. Rakowsky, X. J. Wang, M. Woodle, V. Yakimenko, J. Jagger, V. Sajaev, and I. Vasserman, Phys. Rev. Lett. 86, 5902 (2001).

[7] D. C. Nguyen, H. P. Freund, and W. Colson, Phys. Rev. ST Accel. Beams 9, 050703 (2006).

[8] E. L. Saldin, E. A. Schneidmiller, and M. V. Yurkov, The Physics of Free Electron Lasers (Springer, Berlin, 2000), Chap. 6, p. 265.

[9] H. P. Freund, Phys. Rev. A 27, 1977 (1983).

[10] H.P. Freund, S. Biedron, and S. V. Milton, IEEE J. Quantum Electron. 27, 243 (2000).

[11] H. P. Freund, Phys. Rev. ST Accel. Beams 8, 110701 (2005).

[12] H. P. Freund, L. Giannessi, and W. H. Miner, Jr., J. Appl. Phys. 104, 123114 (2008).

[13] P. A. Sprangle, A. Ting, and C. M. Tang, Phys. Rev. A 36, 2773 (1987).

[14] N. Piovella, Phys. Plasmas 6, 3358 (1999).

[15] V. N. Litvinenko et al., Nucl. Instrum. Methods Phys. Res., Sect. A 304, 463 (1991).

[16] N. A. Vinokurov, Nucl. Instrum. Methods Phys. Res., Sect. A 375, 264 (1996).

[17] H. P. Freund and G. R. Neil, Nucl. Instrum. Methods Phys. Res., Sect. A 475, 373 (2001).

[18] G. R. Neil and H. P. Freund, Nucl. Instrum. Methods Phys. Res., Sect. A 475, 381 (2001). 